\title{
A CONJECTURE OF ORE ON CHAINS IN PARTIALLY ORDERED SETS
}

\author{
SAUNDERS MACLANE
}

In a recent investigation, Ore ${ }^{1}$ has given a form of the JordanHölder theorem valid for an arbitrary partially ordered set $P$. This theorem involves essentially the deformation of one chain into another by successive steps, each step being like that used in the conventional Jordan-Hölder theorem. Ore observes that his first theorem would be slightly easier to apply if it were proved under a weaker hypothesis. The modified theorem runs as follows: ${ }^{2}$

THEOREM. If $P$ is a partially ordered set in which every chain joining two elements is finite, then any complete chain between two elements $b<a$ can be deformed into any other complete chain between the same two elements.

The proof rests on this lemma:

LEMma. Under the hypothesis of the theorem, if $C$ is a complete chain from $b$ to $a$ which cannot be deformed into the complete chain $D$ from $b$ to $a$, there exist in $P$ elements $b^{\prime}<a^{\prime}$ and complete chains $C^{\prime}$ and $D^{\prime}$ from $b^{\prime}$ to $a^{\prime}$ such that $C^{\prime}$ cannot be deformed into $D^{\prime}$ and such that $b \leqq b^{\prime}, a^{\prime} \leqq a$ where either $b<b^{\prime}$ or $a^{\prime}<a$.

Proof. Case 1. $C$ and $D$ have in common the element $e, b<e<a$. Then either $C_{b}^{e}$ cannot be deformed into $D_{b}^{e}$, or $C_{e}^{a}$ cannot be deformed into $D_{e}^{a}$. In these two cases, set $b^{\prime}=b, a^{\prime}=e$ or $b^{\prime}=e, a^{\prime}=a$, respectively.

Case 2. $C$ and $D$ have no elements in common. Since $C$ cannot be deformed into $D$, they cannot together constitute a simple cycle. There will then exist, say, elements $c$ in $C$ and $d$ in $D$ with $b<c<a$, $b<d<a$ and an element $m$ in $P$ with $c \leqq m<a, d \leqq m<a$. Because of the hypothesis that every chain in $P$ joining two elements is finite, there will exist in $P$ finite complete chains $E_{m}^{a}, F_{c}^{m}, G_{d}^{m}$. Then $b$ is joined to $a$ by four complete chains,

$$
\begin{gathered}
C_{b}^{c}+C_{c}^{a}, \quad C_{b}^{c}+F_{c}^{m}+E_{m}^{a}, \\
D_{b}^{d}+G_{d}^{m}+E_{m}^{a}, \quad D_{b}^{d}+D_{d}^{a} .
\end{gathered}
$$

Received by the editors December 15, 1942.

${ }^{1}$ Oystein Ore, Chains in partially ordered sets, Bull. Amer. Math. Soc. vol. 49 (1943) pp. 558-566.

${ }^{2}$ Terminology and notation follow the paper of Ore. 
Since $C$ cannot be deformed into $D$, one of the following three deformations must be impossible:

$$
\begin{gathered}
C_{c}^{a} \rightarrow F_{c}^{m}+E_{m}^{a}, \quad C_{b}^{c}+F_{c}^{m} \rightarrow D_{b}^{d}+G_{d}^{m}, \\
G_{d}^{m}+E_{m}^{a} \rightarrow D_{d}^{a} .
\end{gathered}
$$

In the first case we set $a^{\prime}=a, b^{\prime}=c$; in the second case, $a^{\prime}=m, b^{\prime}=b$; in the third case $a^{\prime}=a, b^{\prime}=d$. In each case we have the conclusion of the lemma.

To prove the theorem, suppose that $P$ were to contain two complete chains $C$ and $D$ joining $b$ to $a$ in such wise that $C$ cannot be deformed into $D$. By induction on $n$, the lemma gives in $P$ elements $a=a_{0} \geqq a_{1} \geqq \cdots \geqq a_{n}$ and $b=b_{0} \leqq b_{1} \leqq b_{n} \leqq a_{n}$ such that for each $i$ either $a_{i-1}>a_{i}$ or $b_{i-1}<b_{i}(i=1, \cdots, n)$, and such that there are complete chains $C_{n}, D_{n}$ joining $b_{n}$ to $a_{n}$ with $C_{n}$ not deformable into $D_{n}$. This construction can be carried on indefinitely, using the axiom of choice to select at each stage a definite pair $a_{n+1}, b_{n+1}$. This produces two sequences of elements $a_{i}, b_{i}$ with

$$
b_{0} \leqq b_{1} \leqq b_{2} \leqq \cdots \leqq \cdots \leqq a_{2} \leqq a_{1} \leqq a_{0} .
$$

Furthermore, the inequality sign holds an infinite number of times here, so that we obtain an infinite chain joining $b=b_{0}$ to $a=a_{0}$, contrary to the hypothesis of the theorem.

HARVARD UNIVERSITY 